\title{
Effect of Sports Club Activities on the Physique and Physical Fitness of Young Japanese Males
}

\author{
Takanori Noguchi ${ }^{1, *}$, Shin-ichi Demura ${ }^{2}$, Shigeru Shimada ${ }^{3}$, Hidetsugu Kobayashi ${ }^{4}$, Shunsuke Yamaji ${ }^{5}$ \& Takayoshi $^{5}$ \\ Yamada $^{5}$ \\ ${ }^{1}$ Fukui University of Technology, Fukui, Japan \\ ${ }^{2}$ Graduate School of Natural Science \& Technology, Kanazawa University, Kanazawa, Japan \\ ${ }^{3}$ Fukui National College of Technology, Fukui, Japan \\ ${ }^{4}$ Sapporo International University, Hokkaido, Japan \\ ${ }^{5}$ University of Fukui, Fukui, Japan \\ *Corresponding author: Department of Industrial Business and Engineering, Fukui University of Technology, \\ Gakuen 3-6-1, Fukui, Fukui 910-8505, Japan. Tel: 81- 776-29-2620. E-mail: t-noguchi@fukui-ut.ac.jp
}

Received: September 30, 2013 Accepted: November 29, 2013 Online Published: December 4, 2013

doi:10.5430/wje.v3n6p27 URL: http://dx.doi.org/10.5430/wje.v3n6p27

\begin{abstract}
This study compared the growth and development of the physique and physical fitness of young Japanese males who belong to sports clubs and those who did not based on longitudinal data obtained over four years. Physique and physical fitness tests were administered to 253 healthy male technical college students four times from the 9 to 12 grade. Subjects were divided into two groups: those who did not belong to an extra-curricular sports club (control group: $C G, n=126$ ), and those who belonged to sports clubs (exercise group: $E G, n=127$ ). Sit-ups, side-stepping, $20 \mathrm{~m}$ shuttle run, $50 \mathrm{~m}$ run and handball throwing tests and a total score were superior in the EG compared to the CG. Differences between the two groups in body mass, Rohrer's index, six physical fitness tests (except for a handgrip and $50 \mathrm{~m}$ run), and the total score increased with grade. The CG was inferior in overall physical fitness to the EG, especially in whole body endurance. It is highly possible that sports club activities significantly and positively affect the growth and development young males during the growth period under 19-years-old and that inactivity inhibits the development of whole body endurance.
\end{abstract}

Keywords: growth and development, longitudinal study, technical college students

\section{Introduction}

Physical fitness reaches its peak between childhood and adolescence. Therefore, it is important to sufficiently develop physical fitness during this period as improvements generally contribute to overall fitness throughout youth (Mikkelsson, Kaprio, Kautiainen, Kujala, Mikkelsson, \& Nupponen, 2006; Beets, Beighle, Erwin, \& Huberty, 2009, Barnekow-Bergkvist, Hedberg, Janlert, \& Jansson, 1998). Furthermore, to increase or maintain physical fitness, it is necessary to perform moderate exercise (Huang \& Malina, 2002). In junior high and high schools in Japan, belonging to school sports clubs greatly contributes toward ensuring sufficient exercise time, and students belonging to sports clubs have the priority usage of athletic facilities and equipment both during and after school (Miller, 2011). In short, the environment necessary for performing exercise has been prepared in schools. In contrast, most students who do not belong to sports clubs cannot access these facilities and equipment easily in schools owing to the fact that they are occupied by club activities. In addition, sports clubs in Japan tend to focus strongly on competitions (Aoki, 2003). Hence, the percentage of students who avoid belonging to sports clubs, or quit clubs to avoid hard practice, is very high (Yokota, 2002).

The above described situation may be different in many European countries such as Germany. In Europe, they have a social sports system that is massive compared with Japan. Hence, students can choose various sports freely (Heinemann, 1999). This difference in social structure may also greatly affect the growth and development of physique and physical fitness of young people. Due to that fact that students who do not belong to sports clubs are 
reported to have a higher risk of developing cardiovascular diseases (Kawabe, Murata, Shibata, Hirose, Tsujioka, Saito \& Saruta, 2000), it is imperative to fully understand the physical fitness characteristics of young people from a perspective of health and preventive medicine (Stone, McKenzie, Welk \& Booth, 1998).

This study aimed to compare the growth and development of physical physique and fitness levels between young Japanese males who belong to sports clubs or not based on longitudinal data obtained over four years.

This study set the following three hypotheses: First, students belonging to sports clubs are superior in physique to those who do not belong to them. Second, students belonging to sports clubs develop superior fitness levels; however, students who do not belong to sports clubs show little change or decreases in physical fitness during their growth. Third, the effects of sports club activities on the development of physical fitness differ according to physical fitness elements.

\section{Method}

\subsection{Subjects}

The subjects in this study included 253 male technical college students among 587 from the first to fourth grade (15-19 years of age) who participated in physical fitness measurement continually for four years. Subjects were divided into one of the following two groups based on a survey of sports club participation: those who did not belong to an extra-curricular sports club, or control group (CG) $(n=126)$, and those who belonged to sports clubs for four years, or exercise group $(\mathrm{EG})(\mathrm{n}=127)$.

Table 1 shows the physique of both groups. Sports clubs of EG were as follows: track and field $(n=6)$, table tennis $(n=8)$, basketball $(n=11)$, rugby $(n=4)$, soccer $(n=12)$, baseball $(n=16)$, volleyball $(n=11)$, badminton $(n=10)$, tennis $(n=9)$, soft tennis $(n=9)$, judo $(n=2)$, kendo(Japanese fencing) $(n=8)$, handball $(n=6)$, softball $(n=6)$, karate $(n=3)$, Shorinji Kempo $(n=5)$, and alpine ski $(n=1)$. Subjects participated in sports clubs two to seven days per week and practiced about two hours daily.

Before measurement, the purpose and procedure of the study were explained in detail to all participants and written informed consent was obtained. This experimental protocol was approved by the Ethics Committee on Human Experimentation of the Faculty of Education, Kanazawa University (No.19-4).

\subsection{Physique Measurement and Physical Fitness Tests}

Height, body mass, and Rohrer's index were selected as indices of physique characteristics. The Ministry of Education, Culture, Sports, Science and Technology (MEXT) physical fitness test was selected to evaluate each subject's physical fitness (The Ministry of Education, Culture, Sports, Science and Technology, 2000).

The physical fitness evaluation consists of eight tests: grip strength (strength), sit-ups (muscle endurance), sit and reach (flexibility), side-stepping (agility), $20 \mathrm{~m}$ shuttle run (whole endurance), $50 \mathrm{~m}$ run (speed), standing broad jump (lower body power), and handball throwing (upper body power). They were performed according to the enforcement method recommended by MEXT for physical fitness tests. Based on the score table provided by MEXT, the records of each test were converted into a scale of $0-10$ and the total score was used as the general physical fitness evaluation value.

The physical fitness test was performed four times from the first grade to fourth grade during regular classes of health and physical education from early April to early June by experienced and expert physical fitness teachers.

\subsection{Statistical Analysis}

Two-way repeated measures analysis of variance (ANOVA) $(2 \times 4$ : participation condition of sports club $\times$ grades $)$ was used to reveal the mean differences for each variable. When a significant interaction or main effect was found, a multiple comparison test was conducted using Tukey's honestly significant difference method. In addition, regression and intercept coefficients were calculated using the means for four years in both groups and their significant differences were tested. $\mathrm{p}<0.05$ was considered to be significant.

\section{Results}

Table 1 shows the characteristics of physique for both groups and Table 2 shows the results of two-way ANOVA (participation condition of sports club $\times$ grade), coefficients and intercept of the regression analysis and their significant test, and the results of significance tests between coefficients and intercepts of both groups. Significance 
was found in a grade factor for all physique variables and results of a multiple comparison test showed that they increase with grade. Regression equation of body mass in both groups and Rohrer's index in EG was significant. Significant differences were found between regression coefficients of body mass and Rohrer's index of both groups.

Table 1: Two-way ANOVA of (participation condition of sports club x grade) and the Regression Analysis of Physique

\begin{tabular}{|c|c|c|c|c|c|c|c|c|c|c|c|c|}
\hline & & \multicolumn{2}{|c|}{ CG $(\mathrm{N}=126)$} & \multicolumn{2}{|c|}{$E G(N=127)$} & & \multirow[b]{2}{*}{ F-value } & \multirow[b]{2}{*}{$p$-value } & \multirow[b]{2}{*}{ Tukey's HSD } & \multicolumn{3}{|c|}{ regression analysis } \\
\hline & & Mean & $\mathrm{SD}$ & Mean & $\mathrm{SD}$ & & & & & & regression & intercept \\
\hline \multirow{4}{*}{$\begin{array}{l}\text { height } \\
\text { (cm) }\end{array}$} & $1 \mathrm{st}$ & 169.1 & 4.67 & 169.5 & 5.34 & $\mathrm{~F} 1$ & 0.40 & 0.51 & CG, EG: $1<2<3,4$ & CG & 0.72 & $168.75 *$ \\
\hline & $2 \mathrm{nd}$ & 170.6 & 5.02 & 171.0 & 5.22 & F2 & 209.40 & $0.00 *$ & & EG & 0.77 & $169.05 *$ \\
\hline & $3 \mathrm{rd}$ & 171.2 & 5.24 & 171.5 & 5.23 & IN & 1.00 & 0.41 & & significant difference & -0.90 & -0.03 \\
\hline & 4 th & 171.3 & 5.28 & 171.9 & 5.30 & & & & & & & \\
\hline \multirow{4}{*}{$\begin{array}{l}\text { body mass } \\
(\mathrm{kg})\end{array}$} & 1 st & 58.6 & 9.57 & 59.0 & 9.31 & $\mathrm{~F} 1$ & 1.40 & 0.24 & CG: $1<2,3<4$ & CG & $1.05 *$ & $57.85 *$ \\
\hline & $2 \mathrm{nd}$ & 60.4 & 9.23 & 61.5 & 8.78 & F2 & 160.50 & $0.00 *$ & EG: $1<2<3<4$ & EG & $1.54 *$ & $57.90 *$ \\
\hline & $3 \mathrm{rd}$ & 61.0 & 9.22 & 62.8 & 8.70 & IN & 7.00 & $0.00 *$ & & significant difference & $-16.86 *$ & -0.03 \\
\hline & 4 th & 61.9 & 8.95 & 63.7 & 8.70 & & & & & & & \\
\hline \multirow{4}{*}{ BMI } & $1 \mathrm{st}$ & 20.5 & 3.46 & 20.5 & 2.79 & F1 & 0.52 & 0.47 & CG: $1,2<3,4$ & CG & 0.18 & $20.35 *$ \\
\hline & $2 \mathrm{nd}$ & 20.8 & 3.45 & 21.0 & 2.57 & F2 & 50.34 & $0.00 *$ & EG: $1<2<3,4$ & EG & $0.33 *$ & $20.25 *$ \\
\hline & $3 \mathrm{rd}$ & 20.8 & 3.41 & 21.3 & 2.42 & IN & 5.24 & $0.00 *$ & & significant difference & -1.07 & 0.03 \\
\hline & 4 th & 21.1 & 3.25 & 21.5 & 2.52 & & & & & & & \\
\hline
\end{tabular}

F1:whether belongs to a sports club, F2: grade, IN: interaction

Table 2: Two-way ANOVA (participation condition of sports club x grade) and the Regression Analysis of Physical Fitness

\begin{tabular}{|c|c|c|c|c|c|c|c|c|c|c|c|c|c|c|}
\hline \multirow[b]{2}{*}{ test } & \multirow[b]{2}{*}{ unit } & \multirow[b]{2}{*}{ grade } & \multicolumn{2}{|c|}{$\mathrm{CG}(\mathrm{N}=126)$} & \multicolumn{2}{|c|}{$E G(N=127)$} & & \multirow[b]{2}{*}{ F-value } & \multirow[b]{2}{*}{$p$-value } & \multirow[b]{2}{*}{ Tukey's HSD } & \multicolumn{3}{|c|}{ regression analysis } & \\
\hline & & & Mean & SD & Mean & SD & & & & & & regression & intercept & \\
\hline \multirow{4}{*}{ grip strength } & \multirow{4}{*}{$\mathrm{kg}$} & 1 st & 40.5 & 6.85 & 41.3 & 7.18 & $\mathrm{~F} 1$ & 3.63 & 0.06 & $\mathrm{EG}: 1<2,3<4$ & CG & $1.28 *$ & 39.55 & \\
\hline & & 2 nd & 42.6 & 6.61 & 44.1 & 7.04 & $\mathrm{~F} 2$ & 93.41 & $0.00 *$ & CG: $1<2,3,4$ & EG & $1.64 *$ & 40.10 & * \\
\hline & & $3 \mathrm{rd}$ & 43.4 & 6.53 & 44.9 & 6.74 & IN & 1.60 & 0.19 & & significant difference & -0.90 & -0.03 & \\
\hline & & 4 th & 44.5 & 6.27 & 46.5 & 6.21 & & & & & & & & \\
\hline \multirow{4}{*}{ sit and reach } & \multirow{4}{*}{$\mathrm{cm}$} & 1 st & 46.0 & 9.18 & 46.7 & 8.62 & $\mathrm{~F} 1$ & 2.27 & 0.13 & EG: $1<2,3<4$ & CG & $0.98 *$ & 45.25 & \\
\hline & & 2 nd & 47.4 & 9.90 & 48.7 & 8.67 & $F_{2}$ & 27.28 & $0.00 *$ & CG: $1<3,4$ & EG & $1.58 *$ & 45.25 & * \\
\hline & & $3 \mathrm{rd}$ & 48.5 & 9.13 & 49.8 & 9.13 & IN & 1.77 & 0.15 & & significant difference & $-16.86 *$ & -0.03 & \\
\hline & & 4 th & 48.9 & 8.97 & 51.6 & 8.73 & & & & & & & & \\
\hline \multirow{4}{*}{ sit-ups } & \multirow{4}{*}{ time } & 1 st & 26.7 & 5.06 & 29.6 & 5.03 & F1 & 39.50 & $0.00 *$ & EG: $1<2,3<4$ & CG & 0.42 & 26.15 & * \\
\hline & & 2 nd & 26.9 & 4.97 & 30.5 & 5.37 & F2 & 28.60 & $0.00 *$ & CG: $1,2<4$ & EG & $0.85 *$ & 28.80 & * \\
\hline & & $3 \mathrm{rd}$ & 27.2 & 5.24 & 31.5 & 4.65 & IN & 4.36 & $0.01 *$ & $1,2,3,4: \mathrm{CG}<\mathrm{EG}$ & significant difference & $-7.84 *$ & -1.72 & \\
\hline & & 4 th & 28.0 & 5.73 & 32.1 & 4.91 & & & & & & & & \\
\hline \multirow{4}{*}{ side-stepping } & \multirow{4}{*}{ time } & 1 st & 52.6 & 5.43 & 56.3 & 4.48 & $\mathrm{~F} 1$ & 61.31 & $0.00 *$ & EG, CG: $1<2<3,4$ & CG & $1.12 *$ & 51.85 & * \\
\hline & & 2 nd & 54.4 & 5.38 & 58.1 & 4.65 & F2 & 82.20 & $0.00 *$ & $1,2,3,4: \mathrm{CG}<\mathrm{EG}$ & EG & $1.37 *$ & 55.20 & * \\
\hline & & $3 \mathrm{rd}$ & 55.7 & 4.80 & 59.8 & 4.12 & IN & 0.81 & 0.49 & & significant difference & $-9.34 *$ & -2.22 & * \\
\hline & & 4 th & 55.9 & 4.92 & 60.3 & 4.29 & & & & & & & & \\
\hline \multirow{4}{*}{ 20-m shuttle run } & \multirow{4}{*}{ time } & 1 st & 76.6 & 17.76 & 90.6 & 19.80 & F1 & 75.67 & $0.00 *$ & EG: $1<2,3,4$ & CG & -0.51 & 76.80 & * \\
\hline & & 2 nd & 75.1 & 15.59 & 94.4 & 19.83 & $\mathrm{~F} 2$ & 3.05 & $0.03 *$ & 1,2,3,4: CG $<\mathrm{EG}$ & EG & 1.21 & 90.75 & * \\
\hline & & $3 \mathrm{rd}$ & 75.7 & 16.00 & 96.0 & 19.85 & IN & 7.30 & $0.00 *$ & & significant difference & $-17.50 *$ & -1.82 & \\
\hline & & 4 th & 74.7 & 15.80 & 94.1 & 20.49 & & & & & & & & \\
\hline \multirow{4}{*}{ 50-m run } & \multirow{4}{*}{ s } & 1 st & 7.9 & 0.59 & 7.7 & 0.45 & $\mathrm{~F} 1$ & 35.92 & $0.00 *$ & EG: $1>3,4$ & CG & -0.40 & 8.00 & * \\
\hline & & 2 nd & 8.0 & 0.57 & 7.6 & 0.47 & F2 & 11.58 & $0.00 *$ & CG: $1,2>4$ & EG & -0.70 & 7.75 & * \\
\hline & & $3 \mathrm{rd}$ & 7.9 & 0.53 & 7.5 & 0.45 & IN & 2.14 & 0.09 & $1,2,3,4: \mathrm{EG}<\mathrm{CG}$ & significant difference & 0.20 & 0.02 & \\
\hline & & 4 th & 7.8 & 0.50 & 7.5 & 0.43 & & & & & & & & \\
\hline \multirow{4}{*}{ standing broad jump } & \multirow{4}{*}{$\mathrm{cm}$} & 1 st & 215.8 & 24.11 & 221.9 & 18.34 & $\mathrm{~F} 1$ & 5.58 & $0.02 *$ & EG: $1<2,3<4$ & CG & 2.98 & 214.20 & * \\
\hline & & 2 nd & 221.2 & 21.15 & 226.2 & 15.69 & F2 & 45.25 & $0.00 *$ & CG: $1<2,4<3$ & EG & $3.11 *$ & 219.25 & * \\
\hline & & $3 \mathrm{rd}$ & 225.2 & 20.84 & 228.5 & 16.85 & IN & 1.86 & 0.13 & & significant difference & $-10.36 *$ & -1.79 & \\
\hline & & 4 th & 224.4 & 22.18 & 231.5 & 17.69 & & & & & & & & \\
\hline & & 1 st & 21.3 & 4.59 & 25.1 & 4.90 & $\mathrm{~F} 1$ & 42.91 & $0.00 *$ & EG: $1,2<3<4$ & CG & 0.60 & 21.00 & * \\
\hline handhal throwing & & 2 nd & 22.6 & 5.00 & 25.9 & 4.87 & $\mathrm{~F} 2$ & 36.55 & $0.00 *$ & CG: $1<2,3,4$ & EG & $0.70 *$ & 24.45 & * \\
\hline . & $m$ & $3 \mathrm{rd}$ & 22.9 & 5.35 & 26.6 & 4.79 & IN & 1.03 & 0.38 & 1,2,3,4: CG<EG & significant difference & -1.89 & -2.68 & * \\
\hline & & 4 th & 23.2 & 5.55 & 27.2 & 4.47 & & & & & & & & \\
\hline & & 1 st & 45.6 & 8.48 & 51.5 & 7.32 & $\mathrm{~F} 1$ & 57.60 & $0.00 *$ & EG: $1<2<3<4$ & CG & $1.41 *$ & 44.45 & * \\
\hline total s sor & noint & 2 nd & 47.5 & 8.25 & 54.5 & 7.24 & $\mathrm{~F} 2$ & 179.70 & $0.00 *$ & CG: $1<2<3,4$ & EG & $2.12 *$ & 49.80 & * \\
\hline - & & $3 \mathrm{rd}$ & 49.0 & 8.29 & 56.5 & 7.06 & IN & 7.30 & $0.00 *$ & $1,2,3,4: \mathrm{CG}<\mathrm{EG}$ & significant difference & $-55.78 *$ & -8.35 & * \\
\hline & & 4 th & 49.8 & 8.35 & 57.9 & 7.11 & & & & & & & & \\
\hline
\end{tabular}


Significant interaction was found in sit-ups and $20 \mathrm{~m}$ shuttle run tests and a total physical fitness score and results of a multiple comparison test showed that the EG was superior to the CG in all grades. In addition, the sit-ups test and a total score increased with grade in both groups; however, the $20 \mathrm{~m}$ shuttle run test was larger in the second to fourth grades than the first grade only in the EG.

A significant main effect in both factors was found in side-stepping, $50 \mathrm{~m}$ run, standing broad jump and handball throwing tests. A multiple comparison test showed that the EG was superior in all tests except for the standing broad jump test to the CG in all grades, and the means of the former two tests increased with grade in both groups. The handball throwing test increased with grade in the EG, but stagnated after the second grade in the CG. No significant difference was found in standing broad jump test between both groups in all grades; however, the mean of both groups increased with grade.

A significant main effect in a grade factor was found in grip strength and sit and reach tests, and their means increased with grade. Regression equations in grip strength, sit and reach, and side-stepping tests, and total score of both groups, and sit-ups, standing broad jump, and handball throwing tests of EG were significant. In addition, significant differences were found between regression coefficients of sit and reach, sit-ups, side-stepping, $20 \mathrm{~m}$ shuttle run, standing broad jump tests, total score, and between intercepts of side-stepping, handball throw tests, and total score in both groups.

\section{Discussion}

In this study, we compared physique and physical fitness between two groups of young Japanese males who belong to sports clubs or not based on longitudinal data over four years. No significant differences were found in height, body mass and Rohrer's index between both groups, and in both groups these values increased with grade; however, body mass and Rohrer's index showed a greater tendency in the EG. In short, it is considered that sport clubs activities affect body mass and Rohrer's index, but do not influence height. Kawabe et al. (2000) reported that the percentage body fat (\%BF) of an exercised sample was significantly lower than that of a control group. Consequently, it is inferred that an increase of body mass in the EG is the result of an increase in muscle volume without an increase of body fat.

Although there were no differences in physique between the EG and CG in the first grade, there were significant differences in physical fitness, and these differences became more pronounced with increasing grade. In addition, most of the students in the EG in the first grade previously belonged to sport clubs during junior high school (between the ages of 12-15 years), whereas many of the CG had never been members of sports clubs. Hence, it is inferred that the above effect showed a difference in physical fitness between both groups in the first grade. In particular, the $20 \mathrm{~m}$ shuttle run test showed a largely different developmental trend between both groups. Whole body endurance is closely related to aerobic ability and develops markedly during 12-15 years if age (Armstrong \& McManus, 2011). It is very likely that students who belong to sports clubs during this period develop more endurance due to exercise stimuli than students who did not belong to sport clubs.

In contrast, it is noted that whole body endurance of the CG developed little with grade in spite of the marked development period of physical functions. Aerobic exercises such as jogging or swimming which stimulate cardiorespiratory function must be intentionally performed because they seldom occur in daily life. Although the age level of 15-19-years-old is the marked development period of physical functions, whole body endurance may not develop unless the individual is regularly receiving endurance exercise stimuli.

In addition, sit-ups (muscle endurance) and side-stepping (agility) tests showed marked difference in the first grade. Unlike whole endurance, both developed after the first grade in both groups; however, the development difference increased with grade. The development of muscle function is fully achieved after 15 years of age (Demura, 2011). Hence, it is inferred that development was also found in the CG. To further develop muscle endurance, continued exercise with a high load is required. However, even young people perform little such exercise in daily life.

The side-stepping test demands that subjects repeat rapid successive movements for 20 seconds. Due to the fact that such movements are performed very little in daily life, it would be expected that little improvement would occur during regular growth and development unless similar tasks were performed intentionally and regularly. Continuous exercise with intensity over a certain level would be necessary to develop whole endurance and muscle endurance.

Although $50 \mathrm{~m}$ run (speed), handball throwing (upper body power), and standing broad jump (lower body power) tests are all related to power, the development process with grade differed according to each test. In short, $50 \mathrm{~m}$ run and handball throwing tests showed a difference between both groups in the first grade and remained it until the 
fourth grade. It is considered that exercise experience during the junior high school period (under 15 years) affected results. In contrast, the standing broad jump test did not show effect of belonging to sports clubs in the first grade, but develop afterwards was and was superior in the EG to the CG. In any case, similar to the other physical fitness elements, it is considered that power development in the EG is larger and it reaches a higher level over time. Although the sit and reach (flexibility) test was similar between both groups at the first grade, EG showed significant improvements afterwards. Athletes increase the ranges of motion of joints through warm-up and stretching to prevent injury (McClure, Balaicuis, Heiland, Broersma, Thorndike, \& Wood, 2007; Malliaropoulos, Papalexandris, Papalada, \& Papacostas, 2004). Hence, it is expected that flexibility among the EG may develop. In the present study, flexibility improved in both the EG and CG, the EG predictably showed superior results. Entering into the second growth phase, muscle mass and bone length in males increase markedly (Armstrong \& McManus, 2011; Shelley \& Peter, 2005). Accordingly, the increase induces an increase of muscle tension; unless athletes perform stretching exercises regularly and endeavor to increase ranges of motion, flexibility is limited (Michael, 2004). Stretching exercises to increase flexibility (Decoster, Cleland, Altieri and Russell, 2005) such as are performed in sports clubs are little performed in daily life. In addition, in physical education in Japan, the duration of teaching was extremely short for the improvement of flexibility (i.e., only $5-10 \mathrm{~h}$ in three years during high school) (Aoki, 2008). Hence, it is considered that the CG developed little flexibility.

Grip strength (static strength) did not show any difference between both groups. Gerodimos et al. (2013) reported that handgrip strength was similar in wrestlers and controls in the younger age groups (i.e., in children and young adolescents). The period of 15- to 19-years-old corresponds to the second growth period in males (Shelley \& Peter, 2005). Handgrip is used frequently in daily life. Therefore, it is not unexpected that grip strength may develop to some extent even among students who did not belong to sports clubs.

It is suggested that belonging to sports clubs until 19 years of age, including junior high school, affects the development and maintenance of physical fitness among Japanese males. Further, students who do not belong to extra-curricular sports clubs display an inferior tendency in overall physical fitness. It has been reported that belonging to sports clubs affects also \%BF, blood pressure, and fat metabolism of young males in addition to physical fitness (Kawabe et al., 2000). A decrease of physical activity is significant problems that lead lifestyle related diseases such as hypertension, diabetes, and hyperlipemia in adulthood (Anderssen, Jacobs, Sidney, et al. 1996). The aforementioned physiological change has a high probability of affecting the health of students who do not participate in sports clubs because it is highly related to whole body endurance which was distinctive in this study.

To solve these problems, in Japan, it will be necessary to proceed at the same time by enriching the social sport system and improving the curriculum content of physical education in schools.

\section{Conclusion}

In conclusion, physique and physical fitness levels of young Japanese male students are greatly affected by belonging to sports clubs for four years between the ages of 15- to 19-years-old. In particular, whole body endurance develops little in students who do not perform sports club activity even during adolescence. In addition, body mass, body bulk (Rohrer's index), flexibility, muscle endurance, agility, speed, and power (jumping, and throwing ability) increase growth and development difference with age between students who belong to sports clubs and those who do not. Performing and continuing exercise in adolescents is very important for encouraging growth and development of their physique and physical fitness.

\section{Acknowledgments}

This work was supported by Fukui National College Technology. The authors thank Enago (www.enago.jp) for the English language review.

\section{References}

Anderssen, N., Jacobs, D.R., Sidney, S., Bild, D. E., Stemfeld, B., Slattery, M. L., \& Hannan, P. (1996). Change and secular trends in physical activity patterns in young adults: a seven-year longitudinal follow-up in the coronary artery risk development in young adults study (CARDIA). American Journal of Epidemiology, 143, 351-362. http://dx.doi.org/10.1093/oxfordjournals.aje.a008749

Aoki, K. (2003). Sport value consciousness of high school athletes and its correlates. Japanese Society of Physical 
Education, 48(2), 207-223.

Aoki, K., \& Kohmura, Y. (2008). Consciousness Survey on Educational Contents Concerning 'Fitness' - Targeting College Students Majoring in Physical Education. Journal of Gymnastics for All, 5, 1-6. [Japanese]. http://dx.doi.org/10.4107/gym.5.1

Armstrong, N., \& McManus, A.M. (2011). Physiology of elite young male athletes. Medicine \& Science in Sports \& Exercise, 56, 1-22. http://dx.doi.org/10.1159/000320618

Barnekow-Bergkvist M., Hedberg G., Janlert U., \& Jansson E. (1998). Prediction of physical fitness and physical activity level in adulthood by physical performance and physical activity in adolescence--an 18-year follow-up study. Scandinavian journal of medicine \& science in sports, $8(5$ Pt 1), 299-308. http://dx.doi.org/10.1111/j.1600-0838.1998.tb00486.x

Beets, M.W., Beighle, A., Erwin, H.E., \& Huberty, J.L. (2009). After-school program impact on physical activity and fitness: a meta-analysis. American Journal of Preventative Medicine, 36(6), 527-537.

Decoster L.C., Cleland J., Altieri C., \& Russell P. (2005). The effects of hamstring stretching on range of motion: a systematic literature review. Journal of Orthopaedic \& Sports Physical Therapy, 35(6), 377-387. http://dx.doi.org/10.2519/jospt.2005.35.6.377

Demura, S. (2011). Health and a sports science lecture (2nd edition). Tokyo, Japan. Kyorinsyoin Press. [Japanese]

Gerodimos V., Karatrantou K., Dipla K., Zafeiridis A., Tsiakaras N., \& Sotiriadis S. (2013). Age-related differences in peak handgrip strength between wrestlers and nonathletes during the developmental years. Journal of Strength and Conditioning Research, 27(3), 616-623. http://dx.doi.org/10.1519/JSC.0b013e318257812e

Heinemann, K. (1999). Sport Clubs in Various European Countries. Schorndorf: Hofmann/Stuttgart: Schattauer.

Huang, Y.C., \& Malina, R.M. (2002). Physical activity and health-related physical fitness in Taiwanese adolescents. Journal of Physiological Anthropology and Applied Human Science, 21(1), 11-19. http://dx.doi.org/10.2114/jpa.21.11

Kawabe, H., Murata, K., Shibata, H., Hirose, H., Tsujioka, M., Saito, I., \& Saruta, T. (2000). Participation in school sports clubs and related effects on cardiovascular risk factors in young males. Hypertension Research, 23(3), 227-32. http://dx.doi.org/10.1291/hypres.23.227

Malliaropoulos, N., Papalexandris, S., Papalada, A., \& Papacostas, E. (2004). The role of stretching in rehabilitation of hamstring injuries: 80 athletes follow-up. Medicine \& Science in Sports \& Exercise, 36(5), 756-759. http://dx.doi.org/10.1249/01.MSS.0000126393.20025.5E

McClure P., Balaicuis J., Heiland D., Broersma M.E., Thorndike C.K., \& Wood A. (2007). A randomized controlled comparison of stretching procedures for posterior shoulder tightness. Journal of Orthopaedic \& Sports Physical Therapy, 37(3), 108-114. http://dx.doi.org/10.2519/jospt.2007.2337

Michael, J.A. (2004). Science of flexibility, Illinois, USA, (pp.53-56), Human Kinetics.

Mikkelsson, L., Kaprio, J., Kautiainen, H., Kujala, U., Mikkelsson, M., \& Nupponen, H. (2006). School fitness tests as predictors of adult health-related fitness. American Journal of Human Biology, 18, 342-349. http://dx.doi.org/10.1002/ajhb.20498

Miller, A. (2011). Beyond the four walls of the classroom, In Wills, D. B. and Rappleye, J. eds., Reimagining Japanese education, Oxford, United Kingdom, (pp.171-191) Symposium Books.

Shelley, L., \& Peter, H. (2005). Longitudinal models of long bone growth during adolescence. American Journal of Human Biology, 17, 731-745. http://dx.doi.org/10.1002/ajhb.20441

Stone, E.J., McKenzie, T.L., Welk, G.J., \& Booth, M.L. (1998). Effects of physical activity interventions in youth. Review and synthesis. American journal of preventive medicine, 15(4), 298-315.

The Ministry of Education, Culture, Sports, Science and Technology.(2000). New physical fitness test. Tokyo, JAPAN, Gyousei. [Japanese]

Yokota, M. (2002). Variation in the motivation and burnout scale with continuation or discontinuation of sports in high school athletes. Research of physical education, 47(5), 427-437. [Japanese] 